01;05

\title{
Магнитообъемные эффекты в сильно коррелированных фазах плутония
}

\author{
(C) А.А. Повзнер, А.Г. Волков
}

Уральский федеральный университет им. Б.Н. Ельцина, Екатеринбург, Россия

E-mail: a.a.povzner@urfu.ru

Поступило в Редакцию 17 января 2018 г.

Рассматриваются термодинамические спиновые флуктуации в сильно коррелированных фазах плутония с обменным и спин-орбитальным взаимодействиями. В $\alpha$ - и $\delta$-плутонии спиновые флуктуации приводят к отрицательному магнитообъемному эффекту, определяемому параметром межмодовой связи и зависящему от особенностей электронной структуры. Показано, что отрицательный коэффициент теплового расширения $\delta$-плутония обусловлен аномально сильным взаимодействием мод спиновых флуктуаций.

DOI: $10.21883 /$ PJTF.2018.16.46480.17217

Для фундаментальных и прикладных исследований важно знать, какие свойства плутония связаны с его электронной структурой, а какие обусловлены ядерно-физическими эффектами, в частности процессами старения.

Важная особенность диаграммы изменения объема в зависимости от температуры для фаз плутония связана с возникновением отрицательного теплового расширения в его $\delta$-фазе (которая существует в температурном интервале от 593 до $736 \mathrm{~K}$ ), причем при переходе между $\alpha$ - и $\delta$-фазами плутония имеет место аномально большое (примерно на 27\%) увеличение объема [1]. До сих пор не удается понять, почему коэффициент теплового расширения (КТР) $\alpha$-фазы является положительным, а КТР $\delta$-фазы - отрицательным. Отметим, что для $\delta$-сплавов плутония с $\mathrm{Ga}$ и $\mathrm{Al}$ наблюдаемый в эксперименте КТР хотя и положителен, но значительно меньше по величине, чем в $\alpha$-фазе.

В рамках спин-флуктуационного подхода [2] было получено, что КТР сплавов плутония имеет достаточно большой отрицательный 
нерешеточный парамагнонный вклад. Однако этот подход неприменим к $\alpha$ - и $\delta$-фазам плутония, в основном состоянии которых имеет место компенсация спиновых и орбитальных магнитных моментов вследствие сильного спин-орбитального взаимодействия $[3,4]$. В $\delta$-сплавах Рu такая компенсация нарушается [3].

Из $a b$ initio исследований электронных спектров ОЦК- и ГЦК-кристаллических структур $\alpha$ - и $\delta$-фаз следует, что плотность $f$-состояний вблизи уровня Ферми заметно возрастает, а ширина зоны соответственно уменышается при переходе от $\alpha$ - к $\delta$-Pu $[3,4]$. Хотя это служит указанием на усиление корреляционных эффектов, данные результаты относятся к основному состоянию плутония и, строго говоря, непригодны для определения объемов и тем более КТР конечнотемпературных термодинамических фаз.

Задачей настоящей работы является исследование спин-флуктуационного механизма магнитообъемных эффектов в сильно коррелированных фазах плутония с учетом ранее развитых представлений о влиянии спин-орбитального взаимодействия на их электронную структуру и магнитное состояние.

Для исследования объемных эффектов в системе сильно коррелированных коллективизированных $f$-электронов $\mathrm{Pu}$ с хаббардовским, хундовским и спин-орбитальным взаимодействиями будем учитывать зависимости интегралов электронных перескоков от объема $(V)$ в модели соответственных состояний Хейне [5]. При этом результаты $a b$ initio расчетов $[3,4]$ спектров $f$-электронов плутония приближенно будем описывать соотношением

$$
\varepsilon_{\mathbf{k}, m, \sigma}^{(f)}=\varepsilon_{\mathbf{k}}^{(f)}(V)-(1-m \sigma /|m|) \Delta_{0},
$$

в котором $\Delta_{0}-$ спин-орбитальное расщепление электронных состояний с ненулевыми значениями орбитального магнитного квантового числа $(m), \sigma= \pm 1-$ спиновое квантовое число.

Можно показать, что вследствие значительных орбитальнозависимых эффектов кулоновских корреляций [3], несмотря на баланс спин-орбитального и обменного взаимодействий, возможно упорядочение спиновых магнитных моментов и возникновение температурных спиновых флуктуаций, отнесенных к отдельным орбитам. При этом свободная энергия рассматриваемой электрон-решеточной системы может быть представлена в виде суммы энергий электронной и магнитной

Письма в ЖТФ, 2018, том 44, вып. 16 
подсистем с учетом изменения объема $(\Delta V)$ и упругой деформации решетки

$$
F=F_{e l}(V, T)+F_{m a g}(V, T)+K(\Delta V)^{2} / 2,
$$

где $K-$ изотермическая сжимаемость.

Более конкретное выражение для свободной энергии электронной и магнитной подсистем записывается на основе данных [6]. При этом сильно коррелированная электронная система рассматривается как система $f$-мультиплетов, расщепленных вследствие спин-орбитального взаимодействия. Последнее осуществляется путем использования результатов расчета плотности $f$-электронных состояний $\alpha$ - и $\delta$-фаз плутония в методе LDA $+U+S 0[3,4]$.

Из условий минимума свободной энергии следует, что спинорбитальная энергетическая раздвижка электронных спектров мультиплетов 5/2 и 7/2 вследствие расщепления электронных термов во флуктуирующих обменных полях зависит от температуры из-за тепловых спиновых флуктуаций

$$
\Delta=\Delta_{0}\left(1-A^{-1} \kappa\left(\left(R_{C} / a_{0}\right)^{-2}+1\right)^{-1}\left\langle\mathbf{M}^{2}\right\rangle_{T} / 3\right) .
$$

Здесь $\left\langle\mathbf{M}^{2}\right\rangle_{T}=B(T / A U)^{2}\left(m^{*} / m\right)\left(\left(R_{C} / a_{0}\right)^{-2}+1\right)^{-2}, m$ и $m^{*}$ - масса и эффективная масса $5 f$-электронов, $B \approx \pi / 4$ и $A \approx 1 / 12-$ параметры функции Линдхарда, $R_{C} \sim(U \chi)^{1 / 2}-$ радиус спиновых корреляций, $\kappa$ - параметр мода-мода $m$-й орбитали, который определяется через вторую полную производную свободной энергии $\left(\kappa=\mathbf{M}_{m}^{-2} d^{2} F /\left(d \mathbf{M}_{m}\right)^{2}\right)$ по среднеквадратичному спиновому моменту орбитали на узле: $\mathbf{M}_{m}^{2}=N_{0}^{-1} \sum_{v}\left((\Delta / U)^{2}+\left\langle\mathbf{M}^{2}\right\rangle_{T}\right)$. При этом плотности электронных состояний для одинаковых значений энергии, противоположные по знаку индекса $m$, различаются только по спиновому квантовому числу $[3,4]$.

Условие минимума свободной энергии приводит к ненулевому значению $\mathbf{M}_{m}^{2}$. Однако, суммируя (3) по $m$, получаем аналогично $[3,4]$ равенство нулю спинового $\left(\sum_{m}\left\langle S_{0, m}^{(z)}\right\rangle=-\sum_{m}(m /|m|) \Delta / U=0\right)$ и орбитального момента на узле. В орбитальных состояниях с локальной намагниченностью возникают спиновые флуктуации с амплитудой $\left\langle\mathbf{M}^{2}\right\rangle_{T}$, величина которой определяется флуктуационно-диссипативной теоремой.

Письма в ЖТФ, 2018, том 44, вып. 16 
Далее, используя термодинамическую связь давления и объема, а также соотношений (1), (2), получаем, что орбитальная зависимость спиновых флуктуаций и намагниченностей орбиталей ведет к магнитообъемному эффекту, который в условиях сильных кулоновских корреляций $(U \chi \gg 1)$ описывается соотношением

$$
\Delta V_{M} / V=-\frac{4 l}{K} \Gamma_{e} \sum_{m}\left((\Delta / U)^{2}+\left\langle\mathbf{M}^{2}\right\rangle_{T}\right)
$$

в котором $l=3(f$-электроны $), \Gamma_{e} \partial \ln \varepsilon(\mathbf{k}, V) / \partial \ln V$ - электронная константа Грюнайзена. При нарушении эффектов компенсации возникает антиферро-, ферро- или сильно парамагнитное состояние (см. работу [3] и ссылки в ней). При этом решающий вклад в магнитообусловленное изменение объема описывается парамагнонным слагаемым [2]

$$
\Delta V_{p m} / V=\frac{1}{K} \Gamma_{p m}(U \chi)^{-1}\left\langle\mathbf{M}^{2}\right\rangle_{T},
$$

малость которого определяется величиной фактора обменного усиления восприимчивости. Вследствие отрицательности парамагнонной константы Грюнайзена $\Gamma_{p m}=\partial \ln X(\mathbf{q}, \omega) / \partial \ln V$ для $\delta$-фазы сплавов $\mathrm{Pu,} \mathrm{как}$ было показано в [2] ( $\left.\Gamma_{p m}=-1.78\right)$, возникает отрицательный магнитный вклад.

Поскольку флуктуации спиновой плотности каждой орбитали возрастают с увеличением температуры, локальная намагниченность каждой орбитали убывает (см. (3)). Это ведет к перемешиванию состояний с $j=5 / 2$ и $7 / 2$ и убыванию среднеквадратичного спинового момента оболочки, что обусловливает отрицательный магнитообъемный эффект (см. (2), (3)).

Поскольку локальная спиновая намагниченность мультиплетов по модулю с температурой убывает, возрастают орбитально обусловленные спиновые флуктуации. При этом, согласно нашим оценкам, температурный рост $\left\langle\mathbf{M}^{2}\right\rangle_{T}$ ведет к уменьшению спин-орбитального расщепления и возникновению отрицательного магнитного вклада в $\operatorname{KTP}\left(\Gamma_{e}=-7 / 3[5]\right)$

$$
\alpha(T)=\frac{4}{K}\left|\Gamma_{e}\right|\left(\Delta_{0}^{2} / U\right) \chi \kappa d\left\langle\mathbf{M}^{2}\right\rangle_{T} / d T .
$$

В соответствии с (5) отрицательная составляющая коэффициента теплового расширения тем больше, чем больше значение параметра 
межмодовой связи. Расчеты по формуле (5), основанные на использовании результатов $a b$ initio моделирования электронной структуры, показывают, что в случае $\alpha$-Рu магнитообъемный эффект должен быть значительно слабее, чем в случае $\delta$-Pu. Согласно нашим оценкам на основе данных $a b$ initio расчетов, отношение параметров межмодовой связи для $\delta$ - и $\alpha$-Pu $\kappa_{\delta} / \kappa_{\alpha} \sim 100$. При этом получаем, что величина магнитного вклада в КТР $\delta$-Рu составляет $\sim 10^{-2} \mathrm{~K}^{-1}$, а для $\alpha$-Pu она равна $10^{-4} \mathrm{~K}^{-1}$.

Согласно зонным расчетам, химический потенциал $\delta$-Pu расположен за пределами псевдощели в области энергий, отвечающих состояниям 5/2 мультиплета, а химический потенциал $\alpha$-Pu расположен в псевдощели, и поэтому имеет место заполнение не только состояний $5 / 2$, но и состояний 7/2. Поэтому намагниченность каждой орбитали $f$-состояний $\delta$-Pu оказывается больше намагниченности каждой орбитали $f$-состояний $\alpha$-Pu. Последнее ведет к различию на $21 \%$ значений (магнитообусловленных) объемов основного состояния $(T=0)$ этих фаз плутония.

Рассмотренные эффекты относятся к так называемым „свежим“ образцам плутония, когда влиянием радиационных эффектов можно пренебречь. В противном случае, скорее всего, происходит нарушение эффектов компенсации магнитных моментов вследствие спинорбитального взаимодействия. Условия, при которых это происходит, требуют отдельных исследований. Кроме того, представляет интерес анализ КТР и магнитообъемных эффектов на основе самосогласованного термодинамического моделирования решеточного ангармонизма и $a b$ initio вычислений начальных объемов в основном состоянии $\delta$ - и других фаз плутония [7,8]. Это позволит более адекватно оценить значения решеточных параметров Грюнайзена и уточнить соотношение величин вкладов магнитной и решеточной подсистем в формирование объемных эффектов и теплового расширения. Представляет также интерес изучение корреляций температурного изменения магнитообъемного эффекта и спин-орбитального расщепления, что требует дополнительных фотоэмиссионных исследований.

Исследования выполнены в рамках задания Министерства образования и науки РФ (контракт 3.9521.2017/8.9).

Письма в ЖТФ, 2018, том 44, вып. 16 


\section{Список литературы}

[1] Lanatá N., Yao Y., Wang C.-Z., Ho K.-M., Kotliar G. // Phys. Rev. X. 2015. V. 5. P. 011008.

[2] Solontsov A., Antropov V.P. // Phys. Rev. B. 2010. V. 81. P. 214402.

[3] Shorikov A.O., Lukoyanov A.V., Korotin M.A., Anisimov V.I. // Phys. Rev. B. 2005. V. 72. P. 024458.

[4] Повзнер А.А., Филанович А.Н., Шориков А.О., Лукоянов А.В., Волков А.Г. // Письма в ЖЭТФ. 2014. Т. 99. В. 11. С. 760-765.

[5] Heine V. // Phys. Rev. 1967. V. 153. P. 673-682.

[6] Повзнер А.А., Волков А.Г., Ноговицына Т.А. // ФТТ. 2017. Т. 59. В. 7. С. $1261-$ 1266.

[7] Yin Z.P., Deng X., Basu K., Yin Q., Kotliar G. // Phil. Mag. Lett. 2014. V. 94. P. $620-628$

[8] Ríos-Ramírez J.J., Rivas-Silva J.F., Flores-Riveros A. // Comput. Mater. Sci. 2017. V. 126. P. $12-21$. 